http://dx.doi.org/10.35381/racji.v5i2.752

\title{
Aplicación del derecho a la motivación en la acción de protección: Sentencias de la Unidad de Familia
}

\section{Application of the right to motivation in protective action: Judgments of the Family Unit}

Gabriel Andrés Galarza-Chullca gabriel.galarza@psg.ucacue.edu.ec Universidad Católica de Cuenca, Cuenca

Ecuador

https://orcid.org/0000-0002-3573-1300

Cecilia Ivonne Narváez-Zurita inarvaez@ucacue.edu.ec

Universidad Católica de Cuenca, Cuenca

Ecuador

https://orcid.org/0000-0002-7437-9880

Juan Carlos Erazo-Álvarez

jcerazo@ucacue.edu.ec

Universidad Católica de Cuenca, Cuenca

Ecuador

https://orcid.org/0000-0001-6480-2270

José Luis Vázquez-Calle

jlvazquezc@ucacue.edu.ec

Universidad Católica de Cuenca, Cuenca

Ecuador

https://orcid.org/0000-0003-4980-6403

Recibido: 13 de abril de 2020

Revisado: 15 de mayo de 2020

Aprobado: 25 de mayo de 2020

Publicado: 14 de junio de 2020 


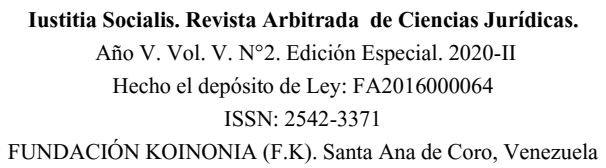

\section{RESUMEN}

La motivación es justificación, exposición de las razones por parte del órgano judicial para demostrar que su decisión es correcta o aceptable, y constituye así, una exigencia del Estado de Derecho. Lo que se pretende en este artículo es demostrar la vulneración de la garantía antes mencionada en las sentencias de acción de protección en perjuicio de las personas que accionan la justicia constitucional. Para esto se utilizó la investigación mixta con énfasis en el enfoque cuantitativo, con carácter descriptivo - explicativo, se especificó las propiedades y características de la garantía constitucional de motivación y cómo debe ser aplicada según parámetros jurisprudenciales de la Corte Constitucional de Ecuador, Ley Orgánica de Garantías Jurisdiccionales y Control Constitucional, así como en la Constitución del Ecuador, con el objetivo de proponer una resolución al Consejo de la Judicatura para la creación de unidades judiciales especializadas en justicia constitucional.

Descriptores: Derecho Constitucional; motivación; administración de justicia; derecho de la familia. (Palabras tomadas del Tesauro UNESCO).

\section{ABSTRACT}

The motivation is justification, exposition of the reasons by the judicial body to demonstrate that its decision is correct or acceptable, and thus constitutes a requirement of the Rule of Law. What is intended in this article is to demonstrate the violation of the aforementioned guarantee in the protective action judgments to the detriment of the people who act the constitutional justice. For this, mixed research was used with emphasis on the quantitative approach, with a descriptive - explanatory nature, the properties and characteristics of the constitutional guarantee of motivation were specified and how it should be applied, according to jurisprudential parameters of the Constitutional Court of Ecuador, Organic Law of Jurisdictional Guarantees and Constitutional Control, as well as in the Constitution of Ecuador, with the aim of proposing a resolution to the Council of the Judiciary for the creation of specialized judicial units in constitutional justice.

Descriptors: Constitutional right; motivation; administration of justice; family law. (Words taken from the UNESCO Thesaurus).

\section{INTRODUCCIÓN}

En Ecuador con la integración al ordenamiento jurídico de la carta magna del año 2008, se sustituyó el esquema de Estado legalista por un modelo donde prima la Constitución, los derechos y la justicia. Esto derivó en cambios importantes de la norma suprema en la 


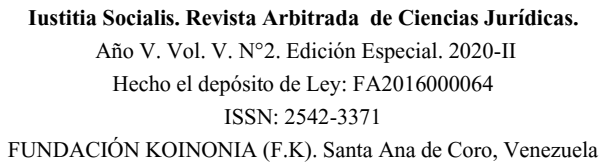

FUNDACIÓN KOINONIA (F.K). Santa Ana de Coro, Venezuela

Gabriel Andrés Galarza-Chullca; Cecilia Ivonne Narváez-Zurita; Juan Carlos Erazo-Álvarez; José Luis Vázquez-Calle

parte orgánica y dogmática debido a avances constitucionales alcanzados en derechos, garantías y justicia que están direccionados sobre todo en favor y respeto del ser humano. Entre estos se encuentra la garantía del debido proceso en la motivación de resoluciones del poder público y en específico cuando las personas accionan un ente de administración de justicia, como por ejemplo en los casos de la acción de protección. Al respecto la (Constitución de la República del Ecuador, 2008, art. 76.7 lit. I) expresa: "en todo proceso en el que se determinen derechos y obligaciones de cualquier orden, se asegurará el derecho al debido proceso que incluirá las siguientes garantías básicas (...) Las resoluciones de los poderes públicos deberán ser motivadas (...)".

Esta garantía constitucional constituye un respaldo a las personas, para que jurídicamente el poder estatal se accione de forma justa y limitada, en procura de siempre racionalizar lo actuado por la Función Judicial en aquellos procesos de garantías jurisdiccionales que inician con el accionamiento por parte de los litigantes.

Esta garantía del debido proceso se analizó y discutió en los Estados constitucionales desde la primera mitad del siglo XX, no obstante, pese a los avances que se ha obtenido, es aún un tema muy controversial, presentándose incluso problemas que se originan por la falta de preparación y especialización por parte de los administradores de justicia sobre regulaciones jurisprudenciales, doctrinales, constitucionales, y, el desarrollo respecto del Sistema Interamericano de Derechos Humanos (SIDH).

En virtud de los antecedentes expuestos se plantea que el problema del presente artículo es: ¿Existe falta de motivación en las sentencias de acción de protección, dictadas en la Unidad Judicial de Familia?

Desde esta perspectiva, el objetivo general de esta investigación, consiste en proponer una resolución al Consejo de la Judicatura para la creación de unidades judiciales especializadas en justicia constitucional, con jueces constitucionales que tengan una especialidad técnica y jurídica en administración de justicia constitucional.

\section{Referencial teórico}

Antecedentes históricos de la garantía de la motivación 
La herramienta constitucional destinada a controlar, limitar, obligar a dar razones al poder judicial es la motivación (Calamandrei, 1960), la misma debe exponer argumentos, razones que el juzgador considera en su sentencia para demostrar que su dictamen es justo, cabal y apropiado, esta garantía no se constituye un simple formalismo que debe cumplir el juez, es una imposición que la debe acatar bajo prevenciones de ser sancionado.

(Atienza, 1991), señala que la motivación significa describir o exponer los acontecimientos, los fundamentos del dictamen (ambiente de hallazgo); así mismo, dar premisas que faculten pensar que un dictamen es preciso o adecuado (ambiente de acreditación). La obligación de motivar nace a partir de la Revolución Francesa en el año 1789, en ambientes de agitación y hervor revolucionario, donde las ideas de las grandes mentes pensadoras y filosóficas del movimiento cultural e intelectual de la llustración, dio paso a un régimen político y social que permitió que la mesocracia se instale en el poder del Estado y para sostenerse en este dominio, el mecanismo que utilizaron fue permitir el control de la ley. (Guzmán, 2014) sobre este dominio de la ley y entendiendo la política revolucionaria de aquella época, menciona que para vigilar esta legalidad se creó un Tribunal de Casación, ya que únicamente existía el magistrado de justicia, quien era representante y puesto por el Rey, el que al distribuir justicia no tenía la obligación de dar argumentos sobre la decisión porque dar justificaciones era visto como un episodio de debilidad política y un ataque al Rey.

En este mismo contexto del Estado legal, el juez aplicaba la ley hecha por el legislador a través de un silogismo jurídico enmarcando los hechos del caso a la ley, de esta manera se entendía que motivaba su decisión. El trato a las cuestiones jurídicas era como simples casos sencillos que fácil se subsumían a las normas legales. 


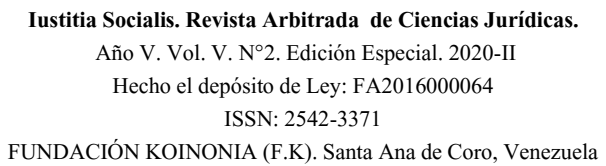

FUNDACIÓN KOINONIA (F.K). Santa Ana de Coro, Venezuela

Gabriel Andrés Galarza-Chullca; Cecilia Ivonne Narváez-Zurita; Juan Carlos Erazo-Álvarez; José Luis Vázquez-Calle

Esta fase de Estado legal va desde 1780 hasta los años de 1945. Después de los Juicios de Núremberg surge el neoconstitucionalismo que da origen a los nuevos Estados constitucionales, donde prevalecen los derechos humanos y la dignidad para que no se vuelva a repetir lo acontecido en el holocausto, anteponiéndose los principios y valores que se van ponderando en pro del ser humano. Las decisiones deben tener un motivo racional, debiéndose justificar de manera argumentada y con regulaciones lógicas.

Desde una mirada constitucionalista, (Sanchís, 2013) destaca la labor que recae en los juzgadores bajo este panorama, al resaltar la exigencia de orientación de argumentos razonables y morales en sus decisiones. Se añade a esto la idea de que el administrador de justicia asume que el lenguaje jurídico no está libre de imprecisiones y confusiones que los actos humanos implican ser tasados y que estos hechos son descubiertos en el proceso, (Taruffo, 2011) menciona que en el Estado de derecho constitucional hay un argumento interno que se deriva del enlace congruente y ecuánime entre las proposiciones fácticas y el derecho, en un esquema razonable de subsunción que guía a justificar la decisión; y el argumento externo que da los entendimientos manifestados en las proposiciones y el derecho aplicado, las razones de la selección y explicación de la norma jurídica utilizada, así como los hechos relevantes para tomar su decisión, predominando la justificación argumentada de juicios de valor en caso de haberlos utilizado, para llegar a la decisión que plasma en una sentencia.

Complementa esto (Guastini, 2014) para quien el argumento interno transmite una conclusión que se infiere de las premisas, aquí se aplica un precepto jurídico, regla, principio o ley. El argumento externo es el acervo de explicaciones aproximadas a ser convincentes, confiables y que apoyen en buena medida la conclusión y la acreditación interna del argumento, teniendo el juez la obligación formal y material de motivar sus resoluciones, independientemente de la causa que se trate.

\section{Garantía constitucional de la motivación}

La Constitución de la República de 2008, en su artículo 76, reconoce las garantías básicas del debido proceso, en el numeral 7 , literal I, señala que las decisiones de las autoridades públicas deben ser motivadas (Constitución de la República del Ecuador, 


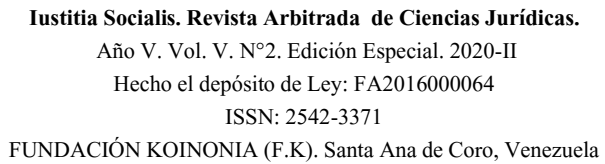

FUNDACIÓN KOINONIA (F.K). Santa Ana de Coro, Venezuela

Gabriel Andrés Galarza-Chullca; Cecilia Ivonne Narváez-Zurita; Juan Carlos Erazo-Álvarez; José Luis Vázquez-Calle

2008 art. 76.7. literal I). De esta forma la Asamblea Constituyente insertó la exigencia constitucional de motivar las sentencias, incluso resoluciones de entes administrativos, agrando un doble efecto a su incumplimiento. El primero es de condición procesal, que es la anulación de la sentencia o resolución; y, el segundo, de carácter administrativo, sancionando al responsable.

La Corte Constitucional del Ecuador (CCE) se pronunció por primera vez en el caso 1212 - 11 - EP de fecha 15 de julio de 2011 sobre la motivación, inclusive desarrolló un test de motivación para verificar que las decisiones cumplan criterios de razonabilidad, lógica y comprensibilidad, conceptos que son de aplicación imperativa. En primer lugar, se debe verificar la articulación de las proposiciones normativo - jurídicas aplicadas al caso, después examinar un segundo parámetro de conexión y solidez entre las normas jurídicas utilizadas y luego comprobar el entendimiento de los argumentos (Corte Constitucional de Ecuador, 2012).

La CCE por medio de la jurisprudencia ha establecido estos elementos que componen la motivación. Al respecto, el máximo organismo de administración de justicia constitucional ha expresado en su sentencia 020-13-SEP-CC que la autoridad que decida debe exponer sus argumentos de manera razonable, lógica y comprensible (Corte Constitucional de Ecuador, 2013).

El análisis de cada uno de estos elementos en la sentencia 0061-14-SEP-CC de la CCE y Ley Orgánica de Garantias Jurisdiccionales y Control Constitucional LOGJ y CC, art. 4 numeral 10 da conceptos claros sobre los elementos de la motivación:

Razonabilidad. - Consiste en enunciar las normas y principios que tengan relación con el proceso o la causa a resolverse, es decir es la fundamentación en derecho. Lógica. - Es la relación entre los hechos y la norma, debe existir un orden lógico entre lo expuesto, es la aplicación correcta del silogismo jurídico.

Comprensibilidad. - La resolución debe ser clara y sencilla, comprensible para cualquier persona, además este parámetro es el resultado de la aplicación correcta de los dos anteriores, y su omisión puede anular los tres parámetros.

(Corte Constitucional del Ecuador, 2014, pág. 8). 
La CCE a más de desarrollar el concepto de motivación y sus elementos, advierte que, si no se motiva un fallo judicial, se vulnera uno de los ejes que conforman el amplio espectro de garantías del debido proceso, ya que al otorgar razones lógicas, suficientes y comprensibles sobre una decisión se demuestra no solo la garantía de la motivación, sino que se permite que los litigantes analicen y entiendan el trabajo argumentativo realizado por el juzgador para resolver el tema sujeto a la justicia, por lo tanto los jueces de todas las ramas deben motivar sus decisiones, incluyéndose dentro de estos a los jueces que tramiten acciones de protección.

\section{La acción de protección como mecanismo de garantía de derechos}

En el Estatuto Federalista Mexicano del año 1957 se conserva un antecedente de la acción de protección, se la conocía como acción de amparo constitucional, y esta acción de la carta mexicana está inspirada en el hábeas corpus de fuente anglosajona, en un principio el amparo protegía la libertad e integridad personal. En América Latina, localizamos el mandato de seguranza en Brasil, la acción de tutela en Colombia, el amparo constitucional peruano y en Chile el recurso de protección (López, 2018).

Esta garantía jurisdiccional se puede rastrear en la Convención Americana de Derechos Humanos o Pacto de San José, ratificado por Ecuador el 12 de agosto de 1977, en su artículo 25 se dispone que el ser humano ostenta su facultad de acceder a recursos sencillos, rápidos y efectivos ante la justicia (Convención Américana sobre Derechos Humanos, Pacto de San José de Costa Rica, 1978, art. 25).

La Declaración Universal de Derechos Humanos el 10 de diciembre de 1948, señala que las personas tienen derecho a acudir a la justicia y presentar procesos diligentes, rápidos que los amparen frente a cualquier acto que quebrante sus derechos legales y constitucionales (Pásara, 2008).

Con toda esta base y para proteger a la ciudadanía de los abusos o negligencias de aquellos que detentan el poder en ejercicio de funciones públicas, e inspirados en posturas similares de otros países del área latinoamericana, los constituyentes incorporaron esta acción que permite reclamar ante la justicia constitucional la vulneración de los derechos garantizados en la Constitución. 


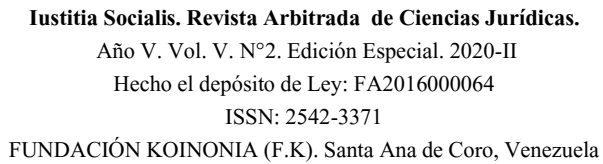

FUNDACIÓN KOINONIA (F.K). Santa Ana de Coro, Venezuela

Gabriel Andrés Galarza-Chullca; Cecilia Ivonne Narváez-Zurita; Juan Carlos Erazo-Álvarez; José Luis Vázquez-Calle

En Latinoamérica la acción de protección recibe denominaciones como amparo, tutela, mandato de seguridad, protección. El nombre que se le dé en las constituciones de los diferentes países a esta garantía jurisdiccional no difiere en su propósito o ideal que es la tutela de los derechos y garantías inalienables de las personas (Fix-Zamudio, 1997). En el capítulo VI artículo 95 de la Constitución Ecuatoriana de 1998 existía la acción de amparo y su objetivo era precautelar que no se vulnere un derecho, en el artículo 88 de la CRE del 2008, la acción de protección tiene condición de garantía jurisdiccional, desarrollada y protectora que la acción de amparo constitucional jamás tuvo. La acción de protección, es un proceso de conocimiento, declarativo y no residual, siendo un salto épico en la protección de los derechos constitucionales del ser humano. El juez constitucional debe ahora declarar la violación del derecho fundamental y reparar las consecuencias; reparación que abarca medidas positivas y negativas, materiales e inmateriales, garantías de no repetición. Esta construcción jurídica consolida esta acción como un mecanismo constitucional útil para la protección de derechos fundamentales.

La acción de protección según la (Constitución de la República del Ecuador, 2008, art. 88) es aplicable a:

1. Los actos u omisiones del poder público, no judicial que vulneren derechos constitucionales;

2. Políticas públicas que no permiten el goce o ejercicio de los derechos y garantías;

3. Los actos u omisiones del prestador del servicio público que viole los derechos y garantías;

4. Los actos u omisiones de las personas naturales o jurídicas del sector privado, en caso de prestación y provocación de daño grave por:

a. Prestaciones públicas impropias o de interés estatal;

b. Prestaciones públicas por encargo o concesiones;

5. Sometimiento o desprotección ante un poderío económico, o conglomerado social o cultural, también religioso o de cualquier otro tipo.

En Ecuador los derechos no solo están escritos en el texto constitucional, sino que gozan de mecanismos como la acción de protección que garantizan el ejercicio de los derechos, 
pudiendo demandar su vulneración a fin de que sean resarcidos los daños que se ocasionen a manera de reparación integral, de esta forma los derechos constitucionales, no quedan en meros enunciados textuales (Silva, 2008).

De acuerdo con criterio de (Bazán, 2010) en el diario ejercicio profesional y en las demandas de acción de protección se debe tomar en consideración lo siguiente:

a) Los jueces constitucionales están facultados para dictar las medidas de reparación integral más adecuadas para resarcir el derecho vulnerado.

b) Aplicación de iura novit curia (dame el hecho, yo te daré el derecho) y sentencias motivadas o inmotivadas.

c) Si la vía constitucional no es la más adecuada para proteger el derecho, el juez debe indicar cuál es la idónea.

\section{Administración de justicia constitucional}

En Ecuador tiene competencia para conocer acciones de protección en primera instancia según la Ley Orgánica de Garantías Jurisdiccionales y Control Constitucional, 2009 “(...) cualquier jueza o juez (...) del lugar donde se origina el acto u omisión (...) que vulnere derechos constitucionales "(...) o donde se producen sus efectos (...)" (Asamblea Nacional, 2009, Art.7) .

Del análisis del artículo anterior se infiere que tener la calidad de juez de primer nivel, es suficiente, para que sea competente en resolver acciones jurisdiccionales, el único límite es el área, o territorio donde se ha pronunciado el hecho o negligencia recurrida o el espacio en el que se producen sus efectos; no hay barrera alguna en cuanto a la materia. La acción de protección sustenta una historia procesal de reclamo por violación de derechos constitucionales en perjuicio de cualquier persona física o jurídica que estime el quebranto de sus derechos garantizados en la Constitución, la misma culmina en una sentencia judicial la mayoría de veces o puede fenecer también por medio de auto definitivo que declare un desistimiento o apruebe un allanamiento (Asamblea Nacional, 2009); en caso de que en la sentencia se declare vulneración de derechos se ordenará la respectiva reparación integral. La sentencia es la decisión final que pone el juzgador a una contienda jurídica. 
La estructura de lo que debe contener una sentencia de acción de protección según la contiene:

1. Antecedentes.

2. Fundamentos de hecho.

3. Fundamentos de derecho.

4. Resolución motivada.

5. Reparación integral del derecho violado.

Es obligación entonces de los jueces, motivar las resoluciones en la acción de protección conforme los criterios desarrollados por la Corte Constitucional y sus precedentes, aplicar la Constitución y la Ley Orgánica de Garantías Jurisdiccionales y Control Constitucional razón por la que el administrador de justicia debe tener conocimientos amplios en esta rama del Derecho para que se garantice el pleno ejercicio de derechos y cumplimiento del marco jurídico.

El análisis crítico de sentencias es el proceso de examinar, detallar, o considerar por separado sus partes para así conocer las características y extraer las conclusiones de los fallos en acciones de protección poniendo en relieve las fortalezas y debilidades de los jueces, si contienen aspectos como test de motivación, los elementos que componen este test y si los jueces ordinarios que administran justicia constitucional sitúan su labor argumentativa en los contextos y fallos constitucionales de la CCE y a partir de esto determinar si están motivadas las resoluciones.

\section{MÉTODO}

El presente estudio transversal presentó un carácter descriptivo - explicativo, por cuanto se especificó origen, concepto y desarrollo de la garantía constitucional de motivación y de la acción de protección, recogidos en libros, leyes y jurisprudencia constitucional, así como en la Constitución de la República del Ecuador. Además, logró un alcance explicativo ya que se estableció la relación de las variables, determinando las causas del fenómeno en estudio (vulneración de la garantía de motivación). La información fue levantada mediante la revisión y análisis de la Constitución de la República del Ecuador, 


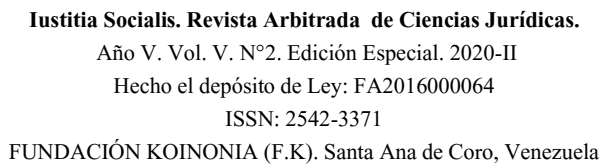

FUNDACIÓN KOINONIA (F.K). Santa Ana de Coro, Venezuela

Gabriel Andrés Galarza-Chullca; Cecilia Ivonne Narváez-Zurita; Juan Carlos Erazo-Álvarez; José Luis Vázquez-Calle

Ley Orgánica de Garantías Jurisdiccionales y Control Constitucional, sentencias y jurisprudencia de la Corte Constitucional Ecuatoriana, libros y artículos científicos publicados en revistas jurídicas indexadas en Dialnet, luris Dictio, Scielo, entre otras.

El tipo de investigación fue mixta, con énfasis en el enfoque cualitativo, ya que en la primera fase se procedió con la revisión de conceptos, evolución histórica, desarrollo, formas de garantía y mecanismos que pueden incidir en la disminución del problema en estudio, mientras que, en la segunda fase se utilizó herramientas cuantitativas como la encuesta para el levantamiento de datos y análisis de sentencias (Sampieri, Collado, \& Lucio, 2014).

Los métodos aplicados en esta investigación fueron: histórico - lógico sustentado a través del tiempo y de manera cronológica, dentro de un orden jurídico constitucional vigente, apoyado por la jurisprudencia y la ley; el analítico - sintético facultó el análisis de fuentes primarias y secundarias, para dotarlas de relevancia jurídica, y así defender el objetivo planteado y sostener las ideas más importantes que motivaron este trabajo de investigación, considerando el ordenamiento constitucional ecuatoriano (Behar, 2008); el método inductivo-deductivo, permitió el razonamiento para establecer generalizaciones respecto a la problemática de la garantía de motivación y su vulneración al momento de emitir sentencias en acciones de protección.

Se procedió con el análisis de 60 sentencias de acción de protección resueltos por la Unidad Judicial de Familia, Mujer, Niñez y Adolescencia del Cantón Cuenca y se aplicó un muestreo no probabilístico por conveniencia considerando los criterios de facilidad, acceso, disponibilidad de personas e intervalo de tiempo.

\section{RESULTADOS}

Una vez obtenida la información a través del análisis de las sentencias de acción de protección y encuestas realizadas sobre el tema evaluación de la garantía de motivación en la acción de protección a profesionales del derecho en libre ejercicio profesional, los resultados serán analizados en el marco del Derecho Constitucional, de manera que se fortalezca el ámbito de aplicación de esta garantía, para evitar la vulneración de la 
motivación por las personas que accionan la justicia constitucional al demandar acciones de protección.

El estudio de investigación fue realizado con fines académicos, en este sentido, el análisis de las sentencias considero el test de motivación desarrollado por la CRE y en la encuesta intervinieron profesionales del derecho en libre ejercicio profesional que han patrocinado acciones de protección, el diseño estuvo estructurado con 60 sentencias de acción de protección y en las encuestas 13 preguntas cerradas y sus posibles alternativas de respuesta.

Entre los principales resultados se determinó:

\section{Unidad Judicial de Familia del Cantón Cuenca}

La garantía de motivación se estudió en las decisiones judiciales de acciones de protección que ha resuelto la Unidad Judicial de Familia, Mujer, Niñez y Adolescencia del Cantón Cuenca divididas en 58 acciones de protección y 2 acciones de protección con medida cautelar desde julio a diciembre de 2019 (figura 1), la principal guía fue el libro de ingreso de causas facilitado por la Coordinación de la Unidad Judicial de Familia, las sentencias analizadas se las obtuvo del sistema SATJE del Consejo de la Judicatura de Ecuador mediante la búsqueda de las resoluciones ingresando de forma manual su numeración, la restricción espacial y temporal fue a sentencias de primera instancia, toda la información que se analizó es pública.

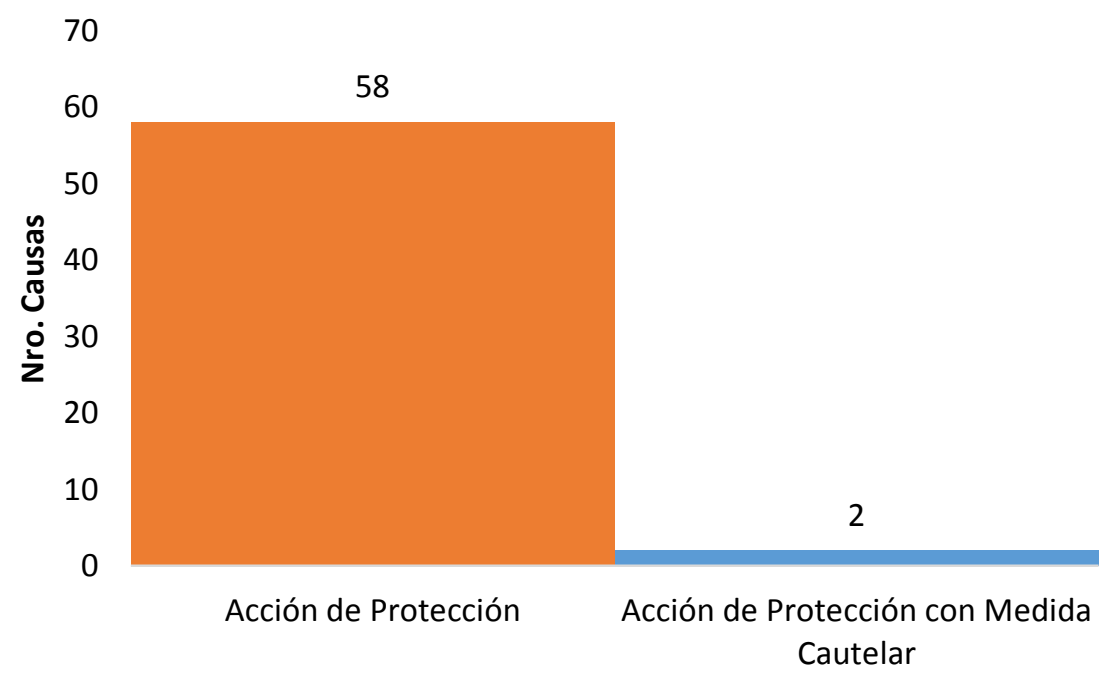


Figura 1. Acciones de Protección resueltas por la Unidad de Familia. Fuente. Libro de ingreso de causas Coordinación Familia Complejo Cuenca

\section{Aplicación del Test de motivación emitido por la Corte Constitucional del Ecuador}

Al analizar las 60 sentencias (figura 2) que forman parte de este estudio, se determinó que el $75 \%$ de jueces ordinarios que administran justicia constitucional en acciones de protección no aplican el test de motivación al expedir sus fallos y un (25\%) sí lo hace.

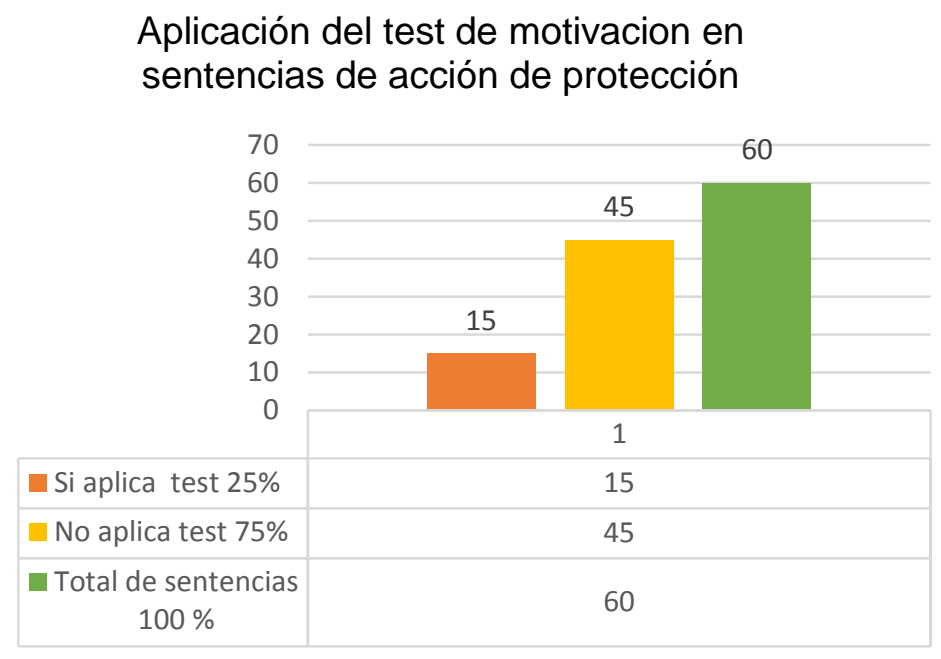

Figura 2. Aplicación de test motivacional a sentencias de acción de protección. Fuente. Sentencias sistema SATJE de la Función Judicial

\section{Razonabilidad, lógica y comprensibilidad de los fallos en acción de protección}

En la tabla 1 se presentan los resultados del análisis de 60 casos de acción de protección resueltos por la Unidad Judicial de Familia, a efectos de determinar si carecen o no verdadero análisis jurídico.

\section{Tabla 1}

Sentencias de Acción de Protección julio a diciembre de 2019

Análisis de Parámetros (test motivación) Sentencias

Contiene

Razonabilidad

Incluye

Lógica
01204-2019-03986; 01204-2019-06715

01204-2019-03986; 01204-2019-06715 


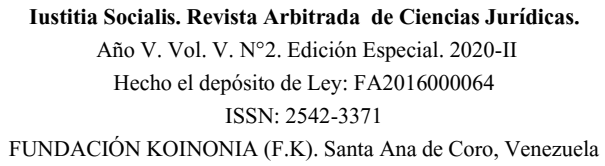

Gabriel Andrés Galarza-Chullca; Cecilia Ivonne Narváez-Zurita; Juan Carlos Erazo-Álvarez; José Luis Vázquez-Calle

Engloba

Entendimiento

No contiene

Razonabilidad

No incluye Lógica

No engloba Entendimiento
01204-2019-03986; 01204-2019-06715

01204-2019-03627; 01204-2019-04229; 01204-2019-04751; 01204-2019-04781; 01204-2019-06043;01204-2019-06633; 01204-2019-01419; 01204-2019-03606; 01204-2019-03821; 01204-2019-04586; 01204-2019-05482; 01204-2019-06328; 01204-2019-06412; 01204-2019-06413; 01204-2019-03817; 01204-2019-03860; 01204-2019-03921; 01204-2019-04170; 01204-2019-04438; 01204-2019-04722

01204-2019-03627; 01204-2019-04229; 01204-2019-04751; 01204-2019-04781; 01204-2019-06043;01204-2019-06633; 01204-2019-01419; 01204-2019-03606; 01204-2019-03821; 01204-2019-04586; 01204-2019-05482; 01204-2019-06328; 01204-2019-06412; 01204-2019-06413; 01204-2019-03817; 01204-2019-03860; 01204-2019-03921; 01204-2019-04170; 01204-2019-04438; 01204-2019-04722; 01204-2019-05170; 01204-2019-05385; 01204-2019-05909; 01204-2019-06523; 01204-2019-03817; 01204-2019-03820; 01204-2019-03821; 01204-2019-03860; 01204-2019-03921; 01204-2019-03947; 01204-2019-04170; 01204-2019-04175; 01204-2019-04229; 01204-2019-04342; 01204-2019-04438; 01204-2019-04465; 01204-2019-04586; 01204-2019-04722; 01204-2019-04751; 01204-2019-04781; 01204-2019-05170; 01204-2019-05173; 01204-2019-05385; 01204-2019-05450; 01204-2019-05482; 01204-2019-05645; 01204-2019-05909; 01204-2019-06043; 01204-2019-06328; 01204-2019-06412; 01204-2019-06413; 01204-2019-06523; 01204-2019-06633; 01204-2019-06643; 01204-2019-06713; 01204-2019-06938; 01204-2019-07058; 01204-2019-07128

01204-2019-03627; 01204-2019-04229; 01204-2019-04751; 01204-2019-04781; 01204-2019-06043;01204-2019-06633; 01204-2019-01419; 01204-2019-03606; 01204-2019-03821; 01204-2019-04586; 01204-2019-05482; 01204-2019-06328; 01204-2019-06412; 01204-2019-06413; 01204-2019-03817; 01204-2019-03860; 01204-2019-03921; 01204-2019-04170; 01204-2019-04438; 01204-2019-04722; 01204-2019-05170; 01204-2019-05385; 01204-2019-05909; 01204-2019-06523; 01204-2019-03817; 01204-2019-03820; 01204-2019-03821; 01204-2019-03860; 01204-2019-03921; 01204-2019-03947; 01204-2019-04170; 01204-2019-04175; 01204-2019-04229; 01204-2019-04342; 01204-2019-04438; 01204-2019-04465; 01204-2019-04586; 01204-2019-04722; 01204-2019-04751; 
01204-2019-04781; 01204-2019-05170; 01204-2019-05173; 01204-2019-05385; 01204-2019-05450; 01204-2019-05482; 01204-2019-05645; 01204-2019-05909; 01204-2019-06043; 01204-2019-06328; 01204-2019-06412; 01204-2019-06413; 01204-2019-06523; 01204-2019-06633; 01204-2019-06643; 01204-2019-06713; 01204-2019-06938; 01204-2019-07058; 01204-2019-07128

Como se puede observar en los resultados de la tabla, las sentencias de acción de protección no contienen motivación, lo único que realizan los juzgadores a-quo es indicar los hechos de forma simple sin expresar razonabilidad, coherencia y lógica de forma holística. En consecuencia, el $75 \%$ de fallos no contiene los parámetros que integran el test motivacional de una sentencia y tan solo un $25 \%$ llegan a tenerlos (figura 3 ).

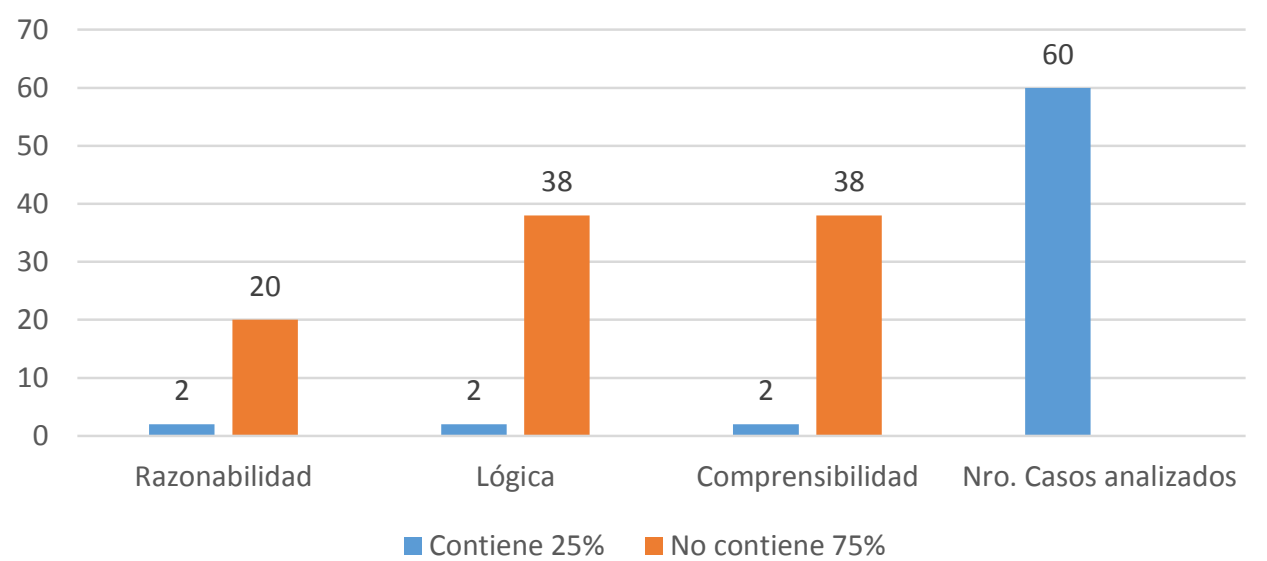

Figura 3. Razonabilidad, lógica y comprensibilidad de sentencias de acción de protección.

\section{Acatamiento de la jurisprudencia de la CCE sobre motivación}

En lo referente al acatamiento y aplicación de la jurisprudencia de la CCE y sobre la garantía constitucional de motivación de sentencias, el 56\% de profesionales encuestados coincidió en que no existe aplicación, mientras que, el $44 \%$ señaló que se aplica de forma incompleta (ver figura 4), estos resultados dejan en evidencia criterios solventes con relación a la existencia de la vulneración de esta garantía, en este sentido, 
resulta trascendental el rol de jueces especializados en garantías jurisdiccionales al momento de decidir en un caso concreto, por cuanto la deficiencia o falta de la motivación en el diario litigar genera inseguridad, y por lo mismo, carencia de racionalización del poder judicial.

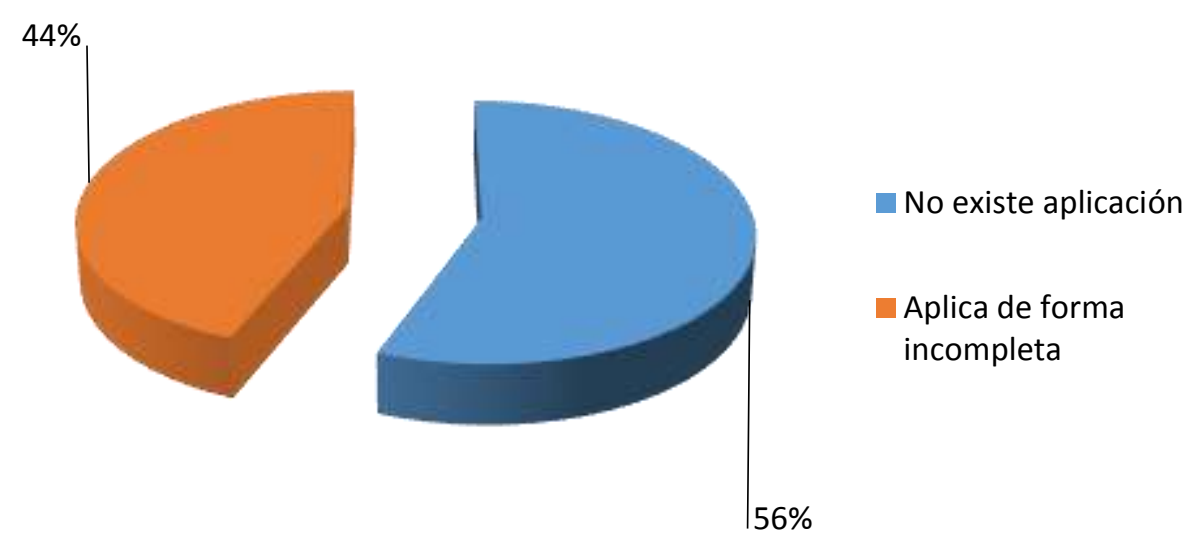

Figura 4. Aplicación de test motivacional a sentencias de acción de protección

\section{Vulneración de la garantía de motivación}

El medio para resolver la problemática a la vulneración de la garantía de motivación en las acciones de protección se sustenta en la posibilidad de requerir del Consejo de la Judicatura la creación de Unidades Judiciales especializadas en justicia constitucional con jueces constitucionales que tengan una especialidad técnica y jurídica para administrar justicia constitucional, motivando sus resoluciones inspirados en el orden jurídico, los abogados encuestados en un $89 \%$ consideran que si es una necesidad la implementación de justicia constitucional especializada frente a un $11 \%$ que dijo no ser 
esencial (ver figura 5).

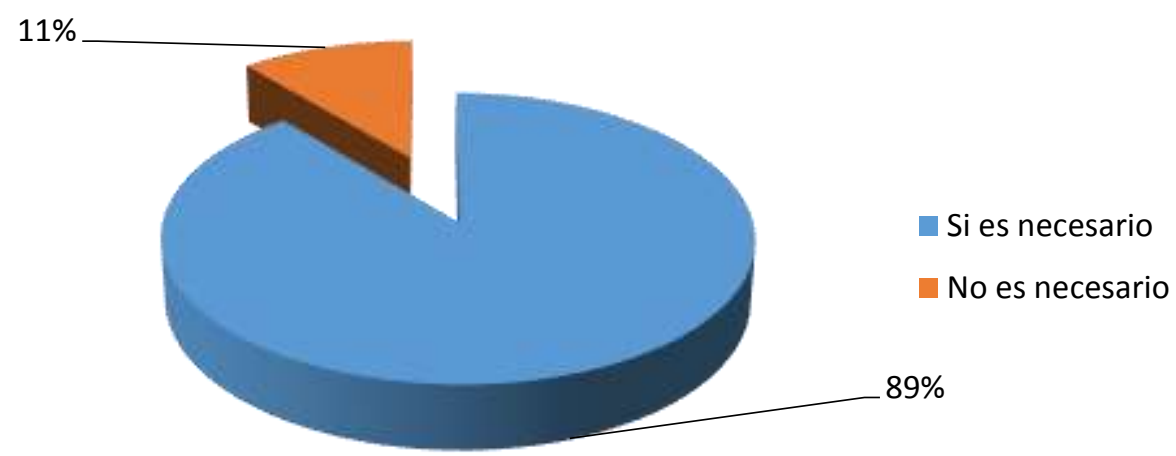

Figura 5. Aplicación de test motivacional a sentencias de acción de protección

De los resultados obtenidos se deduce que los jueces ordinarios que sustancian acciones constitucionales de protección, en su mayoría no motivan y cuando lo hacen esta es incompleta, debido a que no cuentan con especialización en justicia constitucional. Esta apreciación conduce a la investigación a un escenario que tiene que ver con la administración de justicia constitucional y de manera particular con la situación de motivar las sentencias, concretamente sobre los elementos que componen esta garantía como son la razonabilidad, lógica y comprensibilidad, no considerar estos elementos que forman parte de un test desarrollado por la CCE, conlleva a una afectación en la administración de justicia constitucional así como a la vulneración de la garantía constitucional en estudio. Por tanto, se busca mediante esta investigación una propuesta jurídica que proteja a la sociedad que acude a la administración de justicia a fin de no vulnerar sus garantías y a la par permitir el mejoramiento de las resoluciones y conocimientos de la justicia constitucional. 


\section{PROPUESTA}

Conforme a los resultados obtenidos, la problemática es clara y evidencia la vulneración de la garantía del debido proceso sobre motivar las sentencias en acciones jurisdiccionales de protección, por consiguiente, se propone la expedición de una resolución por parte del Consejo de la Judicatura órgano de administración y dirección de la función judicial, para la creación de unidades judiciales especializadas en justicia constitucional, conforme los parámetros establecidos en la figura 6 :

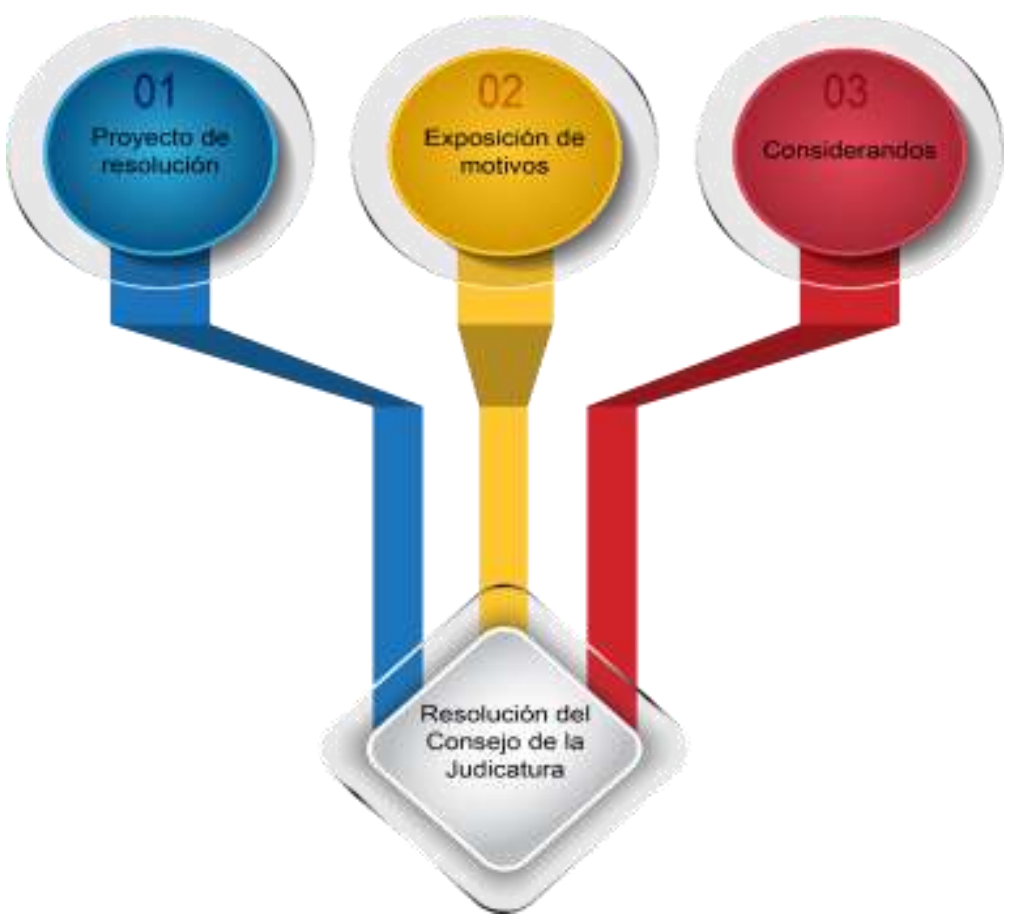

Figura 6. Esquema de propuesta para resolución del Consejo de la Judicatura

\section{Creación de Unidades Judiciales Especializadas en justicia constitucional Exposición de motivos}

Se ha demostrado la vulneración de la garantía del debido proceso en la motivación de sentencias en acciones de protección, por ello, para que no se violen derechos en un proceso constitucional, es importante que el justiciable al accionar garantías jurisdiccionales, acuda a jueces constitucionales que tengan una especialidad técnica y jurídica para administrar justicia constitucional, que motiven sus resoluciones inspirados 


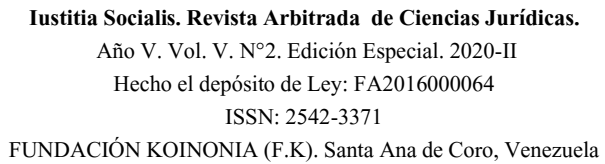

FUNDACIÓN KOINONIA (F.K). Santa Ana de Coro, Venezuela

Gabriel Andrés Galarza-Chullca; Cecilia Ivonne Narváez-Zurita; Juan Carlos Erazo-Álvarez; José Luis Vázquez-Calle

en el orden jurídico, de manera que se establezca una prevalencia mínima de derechos y garantías del debido proceso, para enmarcar así la racionalización del poder judicial a cargo del Estado mediante la motivación de las resoluciones del poder público, siempre con la idea de justicia constitucional especializada como fin garantista de la Constitución. Las acciones jurisdiccionales como la acción de protección en Ecuador deben ser presentadas ante cualquier juez de primer nivel ordinario del lugar donde se origina el acto u omisión o donde se producen sus efectos (Asamblea Nacional, 2009).

De manera simple confluir la condición de juzgador de primer nivel ordinario de jurisdicción cantonal para que pueda conocer y resolver una temática constitucional. Se limita solo en función del territorio, esto es, el lugar donde se ha emitido el acto u omisión recurrida o el sitio donde produce sus efectos; no existe traba alguna en función de la materia que es el eje sustancial de esta propuesta.

Se encuentra una barrera técnica jurídica en el juez ordinario especializado por materia, porque desconoce de justicia constitucional. Si se presenta una acción de protección por vulneraciones a derechos constitucionales ante una destitución de un servidor público, los jueces ordinarios resuelven que esa no es la vía correspondiente ignorando de esta manera precedentes jurisprudenciales obligatorios que un juez especializado puede conocer por especialidad, también suele suceder que la acción jurisdiccional recae por la materia ante el Tribunal Contencioso Administrativo (jueces de primer nivel en esa materia) dicho Tribunal suele ser reservado de opinar sobre los aspectos de derecho administrativo ya que, luego de la acción de protección, le correspondería avocar conocimiento por la vía común de dicha causa, esto deriva en la expedición de un fallo inmotivado.

Al referirse al requisito que el juez constitucional tenga una especialidad técnica y jurídica, es también necesario recalcar que debe utilizar los principios constitucionales en el ejercicio interpretativo y argumentativo. Se pueden crear Unidades Judiciales especializadas con jueces constitucionales de primer nivel sin necesidad de reformar la Constitución, puesto que en ella no se determina qué o cuáles jueces deben conocer las acciones jurisdiccionales, sino que se refiere al ámbito territorial. Debe conformarse el 
grupo de jueces constitucionales por el principio de especialización, para que de esta manera no se vea afectada la administración de justicia constitucional, la garantía de motivación, la seguridad jurídica, la tutela judicial efectiva.

En este contexto, lo que está sucediendo en la actualidad con la justicia constitucional no guarda armonía con la Constitución, se genera una inseguridad jurídica que en esta propuesta de solución se pretende satisfacer. Los jueces ordinarios que conocen garantías jurisdiccionales, no tienen especialidad en esa materia, por ello sus fallos no cumplen el test de motivación emitido por la Corte Constitucional de Ecuador.

Atendiendo a estas consideraciones se presenta la propuesta del proyecto de una Resolución al Consejo de la Judicatura de Ecuador, para la implementación de Unidades Judiciales con especialidad constitucional.

\section{Considerando:}

Que el artículo 341 de la Constitución de la República del Ecuador ordena: "El Estado generará las condiciones para la protección integral de sus habitantes a lo largo de sus vidas, que aseguren los derechos y principios reconocidos en la Constitución, en particular (...) La protección integral (...) a través de sistemas especializados, de acuerdo con la ley;

Que el artículo 76 de la Constitución de la República contiene las garantías del debido proceso, en su numeral 7 , literal I, la cual obliga a que las resoluciones del poder público sean motivadas;

Que el artículo 178 de la Constitución de la República del Ecuador, ordena: "El Consejo de la Judicatura es el órgano de gobierno, administración, vigilancia y disciplina de la Función Judicial. (...)";

Que el articulo 181 numerales 1 y 5 de la Constitución de la República del Ecuador, contemplan: "Serán funciones del Consejo de la Judicatura además de las que determine la ley: 1. Definir y ejecutar las políticas para el mejoramiento y modelización del sistema judicial. (...) 5. Velar por la transparencia y eficiencia de la Función Judicial";

Que el artículo 186 de la Constitución de la República del Ecuador, señala: "El Consejo de la Judicatura determinara el número de tribunales y juzgados necesarios, 
conforme a las necesidades de la población";

Que el artículo 3 del Código Orgánico de la Función Judicial, dispone: "(...) los órganos de la Función Judicial, en el ámbito de sus competencias, deberán formular políticas administrativas que transformen la Función Judicial para brindar un servicio de calidad de acuerdo a las necesidades de las usuarios y usuarios (...)";

Que el articulo 264 numeral 10 del Código Orgánico de la Función Judicial, establece que al Pleno del Consejo de la Judicatura le corresponde: "10. Expedir, modificar, derogar (...) resoluciones de régimen interno, con sujeción a la Constitución y la ley, para la organización, funcionamiento, responsabilidades, control y régimen disciplinario; particularmente para velar por la transparencia y eficiencia de la Función Judicial.";

En ejercicio de las atribuciones establecidas en el artículo 181 de la Constitución de la República del Ecuador y el articulo 264 numeral 10 del Código Orgánico de la Función Judicial,

\section{Resuelve:}

\section{Implementar Unidades Judiciales especializadas en la administración de justicia constitucional a nivel nacional}

Articulo 1.- Implementar el modelo de Unidades Judiciales especializadas en Justicia Constitucional.

Articulo 2.- Las y los jueces de primer nivel ordinarios, que se encuentren sustanciando garantías jurisdiccionales, deberán remitir todos los procesos para que sean sustanciados por las nuevas unidades de justicia constitucional.

Artículo 3.- Las juezas o jueces que integrarán estas unidades judiciales serán elegidos por méritos de concurso y oposición, mediante las directrices emitidas por este pleno.

Articulo 4.- Ofíciese al Ministerio de Finanzas a fin de que asigne los recursos necesarios para la implementación de esta resolución, en base al presupuesto anual remitido el año anterior.

Articulo 5.- Comuníquese a la gestión procesal a fin de que adecue el espacio físico y tecnológico para el funcionamiento de las nuevas unidades judiciales de justicia 
constitucional.

Notifíquese y cúmplase.

Dada en el Distrito Metropolitano de Quito, en la sala de sesiones del Consejo de la Judicatura.

\section{DISCUSIÓN}

En este trabajo se estudió la motivación como garantía constitucional del debido proceso en las sentencias de acción de protección, en su análisis se consideró los elementos que debe contener la motivación de resoluciones y el test emitido por la CCE que contiene los parámetros para que sea aplicada de una forma correcta, de manera que los accionantes de la justicia constitucional estén protegidos en sus derechos al litigar en los tribunales de justicia.

Como consecuencia de este propósito y de acuerdo con la problemática establecida, los resultados del análisis de sentencias y de la aplicación de las encuestas demostraron vulneración a los justiciables en la garantía constitucional de motivación al administrar justicia constitucional en la acción de protección. En este panorama al no existir justicia especializada en materia constitucional, conlleva que jueces ordinarios sin conocimiento técnico jurídico en la materia, emitan sentencias inmotivadas, sin contener razonabilidad, lógica y comprensibilidad, parámetros que se encuentran recogidos en la Constitución, jurisprudencia y criterios de la CCE, tornando ineficaz la justicia constitucional.

Como resultado de esta investigación se propone una resolución por parte del Consejo de la Judicatura que cree unidades judiciales especializadas en justicia constitucional y permita una aplicación técnica en esta materia a fin de que no se vean afectados los derechos de los ciudadanos vulnerando su derecho a obtener un fallo debidamente motivado. En este sentido, el administrador de justicia respete esta garantía básica del debido proceso y sea razonable, lógico y entendible al solucionar un conflicto constitucional. 


\section{FINANCIAMIENTO}

No monetario.

\section{AGRADECIMIENTO}

Al personal de la Unidad Judicial de Familia, Mujer, Niñez y Adolescencia del Cantón Cuenca.

\section{REFERENCIAS CONSULTADAS}

Atienza, M. (1991). Las razones del Derecho. Teorías de la argumentación juridíca; [The reasons of the Law. Theories of legal argumentation]. Madrid: CEC.

Bazán, V. (2010). Derecho Procesal Constitucional Americano y Europeo Tomo II; [American and European Constitutional Procedural Law Volume II]. Buenos Aires, Argentina.

Behar, D. (2008). Metodología de la Investigación;[ Investigation methodology]. México D.F: Shalom. Obtenido de https://n9.cl/v7sb

Calamandrei, P. (1960). Proceso y democracia; [Process and democracy]. Buenos Aires: Ejes.

Carbonell, M. (2007). Teoría del Neo constitucionalismo- El neo constitucionalismo en su laberinto. Ensayos Escogidos, Varios autores, Instituto de Investigaciones Jurídicas-UNAM; [Theory of Neo constitutionalism- Neo constitutionalism in its labyrinth. Selected Essays]. Madrid, España: Trotta.

Constitución de la República del Ecuador; [Constitution of the Republic of Ecuador]. (2008). Asamblea Nacional Constituyente. Montecristi, Manabí, Ecuador: Registro Oficial 449 de 20-oct-2008.

Convención Américana sobre Derechos Humanos (Pacto de San José de Costa Rica);[American Convention on Human Rights (Pact of San José, Costa Rica)]. (1978). Capitulo II Derechos Civiles y Políticos. San José: Nadya Hernández Beltrán.

Corte Consitucional del Ecuador; [Constitutional Court of Ecuador], 227-12-SEP-CC, p. 14 (Corte Constitucional del Ecuador 21 de Junio de 2012). Obtenido de https://cutt.ly/dyPriY4 
Corte Constitucional. (4 de Septiembre de 2013). Sentencia. [Judgment] Recuperado de: https://n9.cl/t4ct. Ecuador.

Corte Constitucional de Ecuador; [Constitutional Court of Ecuador], 020-13-SEP-CC, p.16 (Corte Constitucional de Ecuador 30 de Mayo de 2013). Obtenido de https://cutt.ly/yyPrEwX

Corte Constitucional del Ecuador; [Constitutional Court of Ecuador], 0061-14-SEP-CC, p. 8 (Corte Constitucional del Ecuador 02 de Abril de 2014). Obtenido de https://cutt.ly/WyPr9no

Fix-Zamudio, H. (1997). "La Justicia Constitucional" Revista de Derecho Constitucional No. 1; ["Constitutional Justice" Magazine of Constitutional Law No. 1]. San José, Costa Rica.

Gordillo, D. (2010). La Limitación de la Acción de Protección contra Decisiones Judiciales y su Incidencia en la Indefensión; [The Limitation of the Action of Protection against Judicial Decisions and its Incidence in the Defenselessness]. Quito, Ecuador.

Guastini, R. (2014). Interpretar y argumentar; [Interpret and argue]. Madrid.

Guzmán, L. (2014). Derecho a una sentencia motivada: Integración del debido proceso legal y la tutela judicial efectiva; [Right to a reasoned sentence: Integration of due process of law and effective judicial protection]. Buenos Aires: Astrea.

Ley Orgánica de Garantias Jurisdiccionales y Control Constitucional; [Organic Law of Jurisdictional Guarantees and Constitutional Control]. (2009). Normas generales. Quito, Pichincha, Ecuador: Registro Oficial Suplemento 52 de 22-oct-2009.

López, A. (2018). La acción de protección su eficacia y aplicación en el Ecuador; [Protective action, its effectiveness and application in Ecuador]. Revista Cientifica Dominio de las Ciencias, doi:http://dx.doi.org/10.23857/dom.cien.pocaip.2017.4.1.enero.155-177

Pásara, L. (2008). Declaración Universal de los Derechos Humanos. El uso de los instrumentos internacionales de los Derechos Humanos en la Administración de Justicia, Ministerio de Justicia y Derechos Humanos, Neo Constitucionalismo y Sociedad; [Universal Declaration]. Quito, Pichincha, Ecuador: Ministerio de Justicia y Derechos Humanos.

Sampieri, R. H., Collado, C. F., \& Lucio, M. d. (Abril de 2014). Metodología de la Investigación; [Investigation methodology]. Mc Graw Hill Education, 4. Obtenido de https://n9.cl/65F 


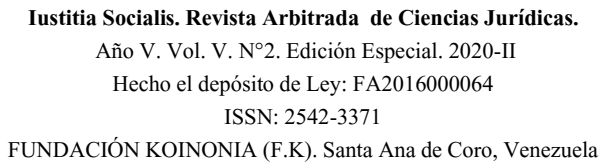

FUNDACIÓN KOINONIA (F.K). Santa Ana de Coro, Venezuela

Gabriel Andrés Galarza-Chullca; Cecilia Ivonne Narváez-Zurita; Juan Carlos Erazo-Álvarez; José Luis Vázquez-Calle

Sanchís, P. (2013). El constitucionalismo de los derechos: Ensayos de folosofia jurídica; [The constitutionalism of rights: Essays of legal folosophy]. Madrid: Trotta.

Silva, P. (2008). Las Garantías de los Derechos ¿Invención o Reconstrucción? Neo constitucionalismo y sociedad; [Rights Guarantees Invention or Reconstruction? Neo constitutionalism and society]. Quito, Ecuador.

Taruffo, M. (2011). La motivación de la sentencia civil; [The motivation of the civil judgment]. Madrid: Trotta.

Vigo, L. R. (2015). Interpretación (argumentación) jurídica en el Estado de derecho; [Legal interpretation (argument) in the rule of law]. Santa Fé: Rubinzal-Culzoni.

\footnotetext{
(C2020 por los autores. Este artículo es de acceso abierto y distribuido según los términos y condiciones de la licencia Creative Commons Atribución-NoComercial-Compartirlgual 4.0 Internacional (CC BY-NC-SA 4.0) (https://creativecommons.org/licenses/by-nc-sa/4.0/).
} 